\title{
Distance Variation Monitoring with Wireless Two-way Interferometry (Wi-Wi)
}

\author{
Bhola Raj Panta, ${ }^{1}$ Kohta Kido, ${ }^{2}$ Satoshi Yasuda, ${ }^{2}$ Yuko Hanado, ${ }^{2}$ Seiji Kawamura, ${ }^{2}$ \\ Hiroshi Hanado, ${ }^{2}$ Kenichi Takizawa, ${ }^{2}$ Masugi Inoue, ${ }^{2}$ and Nobuyasu Shiga ${ }^{2 *}$ \\ ${ }^{1}$ Earthquake Research Institute of the University of Tokyo, \\ 1-1-1 Yayoi, Bunkyo-ku, Tokyo 113-0032, Japan \\ ${ }^{2}$ National Institute of Information and Communications Technology (NICT), \\ 4-2-1 Nukui-Kitamachi, Koganei, Tokyo 184-8795, Japan \\ (Received November 30, 2018; accepted May 21, 2019)
}

Keywords: clock synchronization, distance variation monitoring, millimeter-precision ranging

We demonstrated a simple technique for monitoring distance variation with millimeter precision between two remote sites using off-the-shelf wireless communication modules. The need for the flexible positioning of wireless devices is significantly increasing as more devices are being connected and new services are being developed that require devices to collaborate with one another. We showed that one can monitor distance variation by analyzing the propagation delay of the wireless communication signal between devices. We previously reported a technique for synchronizing clocks with picosecond precision by monitoring the time variation of two rubidium clocks located at remote sites. The precise measurement of the propagation time variation was necessary for the precise synchronization of clocks, and we used this information to estimate the distance with high precision. In a localized situation, our technique makes it easy to implement a millimeter-precision measurement system. Furthermore, it is less complex in terms of system design and can be a low-cost alternative to existing systems that require precise position measurement. We envision that this demonstrated protocol will be implemented in wireless communication chips and microprocessing units.

\section{Introduction}

The history of distance measurement using radio waves goes back to the British Gee and Decca, and American LORAN navigation systems, which used hyperbolic measurement techniques, ${ }^{(1)}$ long before the global positioning system (GPS) became a household name. As the need for distance estimation for both outdoors and indoors grew, ranging systems based on the received signal strength (RSS), the time or time difference of arrival (ToA/TDoA), the angle of arrival (AoA), or the phase of arrival (PoA) metric were extensively researched and made available.

Ranging systems based on some of those techniques were proposed or surveyed by Pahlavan et al., ${ }^{(2)}$ Miesan et al., ${ }^{(3)}$ and Liu et al. ${ }^{(4)}$ Ma et al. ${ }^{(5)}$ compared PoA- and ToA-based ranging

*Corresponding author: e-mail: shiga@nict.go.jp

https://doi.org/10.18494/SAM.2019.2212 
behaviors for radio-frequency identification (RFID) application from the viewpoint of the Cramér-Rao bound. By ray tracing as well as taking measurements in a real environment, they reported that for RFID, PoA-based ranging showed better performance than ToA. Indeed, Exel ${ }^{(6)}$ reported a method based on the carrier phase of IEEE 802.11 wireless local area network (LAN) and its capability was reported to be of sub-centimeter precision. In a more recent paper, Kotaru and Katti ${ }^{(7)}$ also used phase information of the channel state information between Wi-Fi packets to track the position of a virtual-reality headset with sub-millimeter precision.

Precision ranging and positioning systems based on an ultrawide band (UWB) have also been proposed, exhibiting sub-centimeter ${ }^{(8)}$ as well as millimeter precision. ${ }^{(9)}$ More recently, geofencing systems using RSS-based distance estimation with Bluetooth Low Energy, such as Apple's iBeacon and Google's Eddystone, have become available, but they are not geared towards precision ranging. The proposed schemes with higher precision also tend to be more complex for practical implementation. For example, in the method of Exel, ${ }^{(6)}$ the carrier synchronization quality and phase estimation jitter affect the results. For the majority of UWBbased transceivers, despite their simple hardware, most manufacturers are unable to produce inexpensive transceivers. ${ }^{(10)}$

Laser range finders (LRFs) are extensively used for precise distance measurements in construction, manufacturing, land surveying, forestry, and so forth. Interferometric LRFs provide higher accuracy by measuring the interference between incident and reflected beams. Shiga et al. ${ }^{(11)}$ introduced the wireless two-way interferometry (Wi-Wi) technique, which can be considered as an interferometric distance variation measurement system using a wireless signal.

The ubiquitousness of GPS for distance measuring applications has already been mentioned. However, several factors must be considered before implementing GPS-based applications. One of the main concerns is the availability of the GPS signal. This means that GPS-based applications can only be used outdoors. Even outdoors, the required number of GPS satellites may not be visible in dense, canyonlike areas at a given time, degrading the performance of applications or making them unusable.

In the experiment described in this paper, we measured only the phase of the carrier wave, so unlike GPS and LRFs, our experiment does not provide absolute distance measurement but the variation of the distance from an initial position. Wi-Wi can be used indoors as well as outdoors, including in urban, canyonlike environments.

Although distance measurement using the carrier wave phase is not new, the novelty and contribution of our research lie in the fact that with Wi-Wi, it is possible to implement a precision range-monitoring system at any time, indoors or outdoors, using off-the-shelf components, with significantly lower complexity, lower cost, and ease of use. This approach was made possible by the technological improvement of Rb clocks and software-defined radios (SDRs).

\section{System Model}

\subsection{Calculating propagation time using Wi-Wi}

In this section, we explain the method of calculating the propagation time, which is based on the two-way time transfer (TWTT) ${ }^{(12)}$ and two-way carrier phase (TWCP). ${ }^{(13)}$ 
In Fig. 1, the two Wi-Wi systems, site A on the left and site B on the right, have identical setups. Transmitters $\mathrm{X}$ and $\mathrm{Y}$ transmit $2.4 \mathrm{GHz}$ ZigBee radio signals, and the signal from each transmitter is received at two sites: at the local site and at the remote site. We exchange the measured phase values between the two sites by including phase information in the subsequent packets. For a detailed description of the time sequence of this procedure, please refer to Sect. 2.5 of Shiga et al. ${ }^{(11)}$

In the following derivation, we denote time as $T$, as recorded by the timestamp of each attached computer, and the time interval as $t$. Let $t_{c}$ be the time difference between the time of clock $\mathrm{B}, T_{B}$, and that of clock $\mathrm{A}, T_{A}$, i.e.,

$$
t_{c} \equiv T_{B}-T_{A} .
$$

Let $t_{d}$ be the propagation time between the two sites. In this study, transmitter $\mathrm{X}$ at site $\mathrm{A}$ sends a signal to site $\mathrm{B}$ at $T_{X A}$. After a propagation time $t_{d}$, the receiver at site $\mathrm{B}$ receives the signal at $T_{X B}$. The time difference between the reception at site B and the transmission at site A, $t_{B}$, can be expressed as

$$
t_{B} \equiv T_{X B}-T_{X A}=t_{c}+t_{d}
$$

The effect of the propagation time can be measured using the TWTT technique. Hence, similar to the above procedure, we send the transmission time of site B to site A. Transmitter $\mathrm{Y}$ at site $\mathrm{B}$ sends the signal to site $\mathrm{A}$ at $T_{Y B}$. After a propagation time $t_{d}$, the receiver at site $\mathrm{A}$ receives the signal at $T_{Y A}$. The time difference between the reception at site $\mathrm{A}$ and the transmission at site $\mathrm{B}, t_{A}$, can be expressed as

$$
t_{A} \equiv T_{Y A}-T_{Y B}=-t_{c}+t_{d}
$$

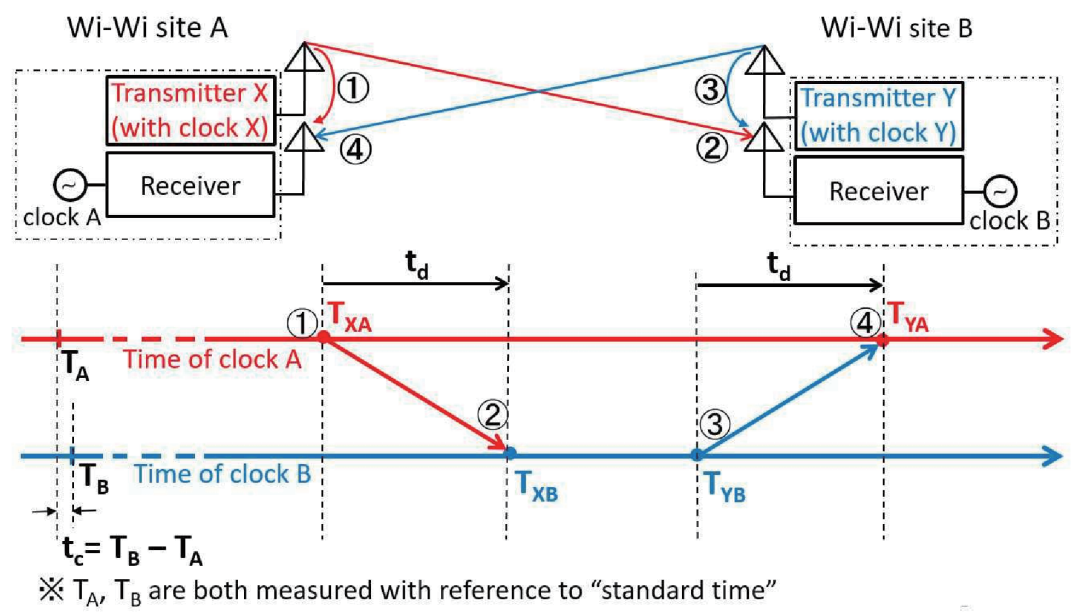

Fig. 1. (Color online) System model of Wi-Wi. The upper half shows the setup of the components and the lower half shows the time sequence. 
Note that the transmission times $T_{X A}$ and $T_{Y B}$ are the reception times measured at sites A and B with clocks $\mathrm{A}$ and $\mathrm{B}$, respectively. We assume that the variations in $t_{c}$ and $t_{d}$ during the roundtrip communication are negligible. Equations (2) and (3) are valid even when there exists frequency difference on the clocks. As one repeats the measurement of $t_{c}$, its value changes at a constant rate with frequency difference. In order to monitor the clock difference $t_{c}$ correctly, we need to ensure that the clock difference measured via the phase is less than $\pi$ between two consecutive measurements. For that purpose, we used a high-precision reference clock to ensure that the clock phase difference is sufficiently small between two measurements (it does not need to be zero).

Adding Eqs. (2) and (3), we obtain

$$
t_{d}=\frac{t_{A}+t_{B}}{2} .
$$

\subsection{Calculating distance using carrier phase}

Because Wi-Wi uses the TWCP in this experiment, we rewrite Eq. (4) using the phase and derive the distance $l_{d}$ between the two sites as

$$
l_{d} \equiv c \cdot t_{d}=-\left(\frac{\varphi_{A}+\varphi_{B}}{2 \pi}+K\right) \frac{\lambda}{2},
$$

where $c$ is the speed of light, $\lambda$ is the wavelength of the carrier wave, $\varphi_{A}$ is the phase advance of the transmitted signal after its propagation from $\mathrm{B}$ to $\mathrm{A}$, and $\varphi_{B}$ is the phase advance of the transmitted signal after its propagation from A to B. Note that the negative sign on the right side is due to the definition of $\varphi_{A}$ and $\varphi_{B}$, according to their measurements at $\mathrm{A}$ and $\mathrm{B}$, respectively. The transmitted signal at $t=0$ is received and measured with reference to the receiver's phase at $t=t_{d}$. As the distance $l_{d}$ is increased, the measured phase of the transmitted signal is shifted negatively. The measured phase can only be obtained modulo $2 \pi$ and $l_{d}$ can only be obtained modulo $\lambda / 2$. The integer ambiguity $K$ must be determined separately to obtain the absolute value of $l_{d}$. However, by measuring $\varphi_{A}$ and $\varphi_{B}$ repeatedly, the variation from the initial measurement can be tracked using the unwrapped phase when the variations in $\varphi_{A}$ and $\varphi_{B}$ between consecutive measurements are guaranteed to be smaller than $\pi$. This principle has been demonstrated by Fujieda et al. ${ }^{(13)}$

The Wi-Wi system (Fig. 1) is configured with four clocks. The goal here is to compare clocks A and B by eliminating the phase difference between the transmitter's built-in clock (clock X or Y) and the receiver clock at the same site (clock A or B). Below, we describe the procedure.

The phase of clock $\mathrm{B}$ with respect to that of clock $\mathrm{A}, \varphi_{B}$, is measured using transmitter $\mathrm{X}$, and the phase of clock $\mathrm{A}$ with respect to that of clock $\mathrm{B}, \varphi_{A}$, is measured using transmitter Y. They are expressed as 


$$
\begin{gathered}
\varphi_{B} \equiv \varphi_{X B}-\varphi_{X A}, \\
\varphi_{A} \equiv \varphi_{Y A}-\varphi_{Y B},
\end{gathered}
$$

where $\varphi_{X B}$ denotes the phase of clock $\mathrm{X}$ received at site $\mathrm{B}$ and $\varphi_{X A}$ denotes the phase of clock $\mathrm{X}$ received at site A. Similarly, $\varphi_{Y A}$ denotes the phase of clock Y received at site A and $\varphi_{Y B}$ denotes the phase of clock $\mathrm{Y}$ received at site $\mathrm{B}$. Although the phases of clocks $\mathrm{X}$ and $\mathrm{Y}$ have no correlation with the rubidium clocks owing to their inaccuracies, $\varphi_{B}$ and $\varphi_{A}$ reflect the phase difference between clocks $\mathrm{A}$ and $\mathrm{B}$, and the distance variation between sites $\mathrm{A}$ and $\mathrm{B}$, including the initial offset. The variation in distance is calculated using Eq. (5).

\section{Experimental Setup}

Figure 2 shows a schematic diagram of the setup for an experiment to demonstrate distance variation monitoring using the system described in Sect. 2. The experiment was conducted in an office with several pieces of furniture and server cabinets.

The two sites A and B were separated by about $40 \mathrm{~cm}$. They had an identical setup and each site consisted of a wireless communication module (ZigBee), an SDR with two receivers, and a rubidium clock. The specifications of each equipment are summarized in Table 1.



Fig. 2. (Color online) Schematic diagram of the experimental setup.

Table 1

Specifications of items used in the experiment.

\begin{tabular}{lc}
\hline Item name & Specification/Model/Manufacturer \\
\hline ZigBee module & $\begin{array}{c}\text { Freq.: } 2.4 \mathrm{GHz}, \text { Power output: } 2.5 \mathrm{dBm} \\
\text { Model: TWE-Lite-USB, } \\
\text { TOCOS (MONOWIRELESS) }\end{array}$ \\
\hline Rubidium clock & SIM940, Stanford Research Systems \\
\hline Software-defined radio & USRP-2942, National Instruments \\
\hline Personal computer (PC) & PXIe-1071, National Instruments \\
\hline
\end{tabular}


The ZigBee modules were connected to a PC through USB cables. The SDRs were referenced to $10 \mathrm{MHz}$, generated by the rubidium clocks, through coax cables. The PC and SDRs were connected by PCI Express (PCIe) cables. Separate channels were assigned to each ZigBee module at the two locations to avoid channel confusion. Each SDR had two receiving channels: channel 1 received the signal from the transmitter at the local site and channel 2 received that from the remote site. ZigBee communication is based on the IEEE 802.15.4 standard, which uses offset quadrature phase-shift keying (O-QPSK).

Transmitters $\mathrm{X}$ and $\mathrm{Y}$ transmitted the signals alternately at a rate of approximately 10 times per second. The SDRs digitized the baseband in-phase (I) and quadrature (Q) 16-bit signals at a rate of 4 megasamples/s and the signals were transferred to the PC via the PCle cable. The PC decoded the signals, symbol by symbol by taking the correlation with the 16 possible chip value sequences. The phase was measured for each symbol and the average of all the unwrapped symbol phases was recorded, which was used to represent the phase of each packet. To demonstrate distance variation monitoring, we changed the position of the antennas of site B by moving the bundled antennas attached to a stepper motor, whose step size was $1 \mu \mathrm{m}$.

A conceptual diagram of the antenna distance variation is shown in Fig. 3. Measurements were taken at distances of (b) 5, (c) 7, and (d) $8 \mathrm{~mm}$ from (a) the initial position. Then, the measurement direction was reversed as illustrated in the figure.

\section{Results}

Figure 4 shows the measurement results. We measured the antenna distance variation over a 6 min interval. The clock difference, plotted in red in the left figure, varied by about 1 ns over the interval, which is reasonable for rubidium clocks. The timing and distance of the antenna movements are indicated in the magnified plot of $t_{d}$, in the right figure.

In our previous paper, ${ }^{(1)}$ we reported a standard deviation of $2.2 \mathrm{ps}$ in propagation time measurement when the antennas were stationary. This means that we can monitor the variation in distance between the antennas with $1 \mathrm{~mm}$ precision since the wireless signal travels about 1



Fig. 3. (Color online) Conceptual diagram of the antenna distance variation. (b), (c), and (d) represent distances of $5 \mathrm{~mm}$, an additional $2 \mathrm{~mm}$, and an additional $1 \mathrm{~mm}$ from the previous position, respectively. The distance was varied using a stepper motor. 




(a)

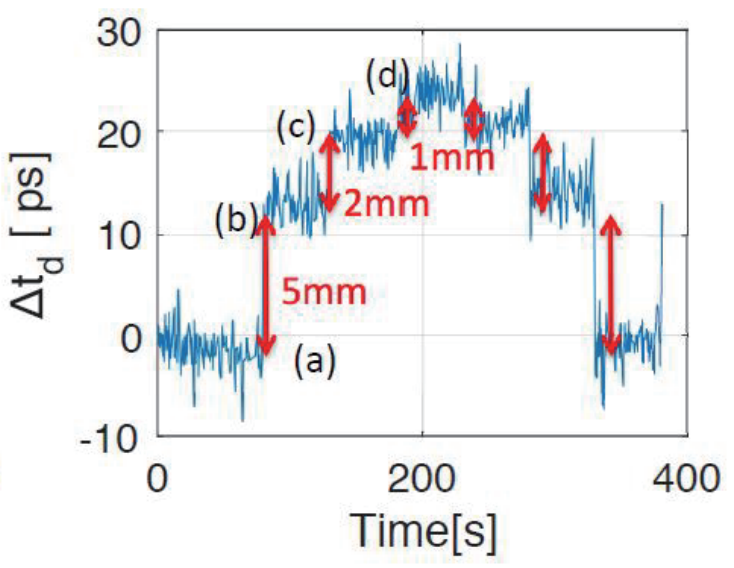

(b)

Fig. 4. (Color online) Experimental results of Wi-Wi. (a) Variation of clock, $\Delta t_{c}$, and variation in propagation time, $\Delta t_{d}$, over 6 min interval. (b) Magnified plot of $\Delta t_{d}$.

$\mathrm{mm}$ in $0.3 \mathrm{ps}$. When we changed the distance by 5,2 , and $1 \mathrm{~mm}$, the propagation time changed by 17,7 , and 3 ps, respectively.

We took the average of the symbol phases over the whole packet to represent the packet phase, resulting in a peak-to-peak deviation of about $10 \mathrm{ps}$, as shown in Fig. 4(b). This 10 ps peak-to-peak deviation is consistent with the previously mentioned standard deviation $1 \sigma$ being $2.2 \mathrm{ps}$. Assuming a normal distribution, data is distributed over $\pm 3 \sigma$, which is 13 ps. If one needs to improve the standard deviation by a factor of 2 and needs to measure the distance in $1 \mathrm{~s}$, one can repeat the measurements four times in $1 \mathrm{~s}$ and take the average over four measurements. Between packets, we used unwrapping to calculate $t_{c}$ and $t_{d}$, as mentioned in the previous section. The unwrapping worked well because the distance variation between adjacent measurements was much smaller than $6 \mathrm{~cm}$, which corresponds to a phase of $\pi \mathrm{rad}$. This method can measure the distance variation only when the variation between adjacent measurements is guaranteed to be smaller than $\lambda / 2$. Note that $t_{c}$ must be continuous and therefore the stability of the reference clock needs to be much higher than $0.5 \mathrm{~ns}$ (corresponding to $\pi \mathrm{rad})$ during the measurement interval $(0.1 \mathrm{~s})$.

\section{Discussion and Conclusion}

In this study, we demonstrated that the proposed Wi-Wi system can monitor the variation in the distance between two antennas with millimeter precision. The core idea of measuring the distance variation between two points is to monitor the phase variation of transmitted signals in both directions. As long as one can monitor the phase of the transmitted carrier wave, Wi-Wi can achieve distance variation measurement. We can apply the method described in this paper to any commercial communication device. So far, we have carried out tests using $2.4 \mathrm{GHz}$ ZigBee, Bluetooth Low Energy, and $920 \mathrm{MHz}$ modules.

Owing to the development of integrated circuits, many wireless communication chips 
digitize the I and Q signals of the carrier wave and process the signal digitally to decode the payload. We envision that phase and Wi-Wi measurements can be embedded in wireless chips and microprocessor units, providing opportunities for the extremely low cost and easy implementation of synchronization and distance measurement using any wireless communication scheme.

The Wi-Wi system is based on interferometric phase measurements of the carrier wave. In this regard, our technique closely resembles the principle of interferometric LRFs. However, the LRFs require very precise beam alignment, and in practical situations, if the reflector of an LRF is moved, setting up and retuning can be cumbersome. Also, if the reflector is on an inclined surface, the measurement may not be accurate. On the other hand, because we used radio waves, and since radio wave propagation is broad by nature, there is no need for precision beam alignment, thereby making the Wi-Wi system easy to use. In a localized environment such as a construction site, surveying for distance measurement to place markers is critical but often carried out in an almost chaotic environment. Therefore, the ability to set up and use a measuring system on-the-fly is highly desirable.

One of the promising applications of propagation measurement using Wi-Wi is monitoring the distance variation between two points. For example, one can use this technique to monitor small changes $(\sim \mathrm{mm})$ in the length or tilt of infrastructure such as towers and bridges. Another interesting application could be atmospheric remote sensing by monitoring the integrated index of refraction (IIR) of air. Using Wi-Wi, the variation in propagation delay, which is mainly due to water vapor, can be estimated with picosecond precision.

In general, it is necessary to calibrate the measured phase for the characteristic of the antennas. In our experiment, we used dipole antennas and the measured phase depended on the relative antenna angle. We did not calibrate for the antenna angle because we kept the antenna angle constant as we changed the distance. In applications where the antenna angle changes, the characteristics of the antenna need to be considered for correct distance variation measurement.

The proposed method is conceptually simple and is attractive because of its low complexity and straightforward setup to carry out experiments. The current system requires rigorous evaluation in multipath environments before its practical use. We believe that developing appropriate measures for a multipath environment will lead to its wider adoption in academic research as well as in industry.

\section{Future Development}

The prototype version we tested used software radio (USRP) and required AC power, and the overall setup is not conductive to mobile use. The miniaturization of the system is already underway and we have fabricated a module with all the required components (high-precision oscillator, TX/Rx and I/O interfaces), a prototype of which is currently being tested.

\section{Acknowledgments}

This work was supported by the JST PRESTO program (Grant No. JPMJPR14D5) and NICT. We thank Ryuichi Ichikawa of NICT for his insightful comments. 


\section{References}

1 D. H. C. Scholes: J. Br. Inst. Radio Eng. 12 (1952) 595. https://doi.org/10.1049/jbire.1952.0059

2 Y. K. Pahlavan, X. Li, and J. Makela: IEEE Commun. Mag. 40 (2002) 112.

3 R. Miesen, F. Kirsch, P. Groeschel, and M. Vossiek: IEEE Int. Conf. Wireless Information Technology and Systems (ICWITS) (IEEE, 2012).

4 H. Liu, H. Darabi, P. Banerjee, and J. Liu: IEEE Trans. Syst. Man Cybern., Part C Appl. Rev. 37 (2007) 1067. https://doi.org/10.1109/TSMCC.2007.905750

5 Y. Ma, K. Pahlavan, and Y. Geng: Proc. IEEE 25th Annu. Int. Symp. Personal, Indoor, and Mobile Radio Commun. (PIMRC) (IEEE, 2014)

6 R. Exel: Proc. IEEE Wireless Commun. Networking Conf. (WCNC) (IEEE, 2013).

7 M. Kotaru and S. Katti: Proc. IEEE Conf. Computer Vision and Pattern Recognition (CVPR) (July, 2017). https://doi.org/ 10.1109/CVPR.2017.286

8 A. M. Petroff: Proc. USNC-URSI Radio Science Meeting (2015) 212.

9 C. Zhang, M. J. Kuhn, B. C. Merkl, A. E. Fathy, and M. Mahfouz: IEEE Trans. Microwave Theory Tech. 58 (2010) 9. https://doi.org/10.1109/TMTT.2009.2035945

10 A. Alarifi, A. Al-Salman, M. Alsaleh, A. Alnafessah, S. Al-Hadhrami, M. A. Al-Ammar, and H. S. AlKhalifa: Sensors 16 (2016) 707. https://doi.org/10.3390/s16050707

11 N. Shiga, K. Kido, S. Yasuda, B. Panta, Y. Hanado, S. Kawamura, H. Hanado, K. Takizawa, and M. Inoue: IEICE Commun. Express 6 (2016) 77. https://doi.org/10.1587/comex.2016XBL0181

12 D. W. Hanson: Proc. 43rd Annu. Symp. Frequency Control (May-June, 1989). https://doi.org/10.1109/ FREQ.1989.68861

13 M. Fujieda, T. Gotoh, F. Nakagawa, R. Tabuchi, M. Aida, and J. Amagai: IEEE Trans. Ultrason. Ferroelectr. Freq. Control 59 (2012) 2625. https://doi.org/10.1109/TUFFc.2012.2503 